\title{
Special issue of selected papers from the Joint 6th Meeting of the European Section and 7th Meeting of the North American Section of the International Academy of Cardiovascular Sciences: "Cardiometabolic Diseases: How New Research May Lead to New Cardioprotective Therapy" (11-14 September 2019, Serbia). Part 1: The North American Section
}

\author{
Guest Editors \\ Dragan Djuric \\ University of Belgrade, Faculty of Medicine \\ Institute of Medical Physiology "Richard Burian" \\ str. Visegradska 26/2, Belgrade, 11000 Serbia \\ Vladimir Jakovljevic \\ University of Kragujevac, Faculty of Medicine \\ Department of Physiology \\ Svetozara arkovića 69, Kragujevac \\ 34000 Serbia \\ Danielle Jacques \\ University of Sherbrooke \\ Department of Anatomy and Cell Biology \\ Faculty of Medicine and Health Sciences \\ 2500, boul. de l'Université, Sherbrooke \\ QC J1K 2R1, Canada
}

